\title{
Zweidimensionale Verzahnungen für lagerlose reluktante Rotations-Linear-Motoren
}

\author{
A. Schleicher, R. Werner
}

\begin{abstract}
Der lagerlose reluktante Rotations-Linear-Motor ist ein neuartiger Antrieb und ermöglicht unabhängig voneinander sowohl magnetisches Schweben als auch Translation und Rotation eines ferromagnetischen Rotors. Zusammen mit der einfachen und robusten Bauweise in Form eines kompakten Aktuators mit Doppelstator ermöglicht dieser Motor ein großes Spektrum an neuen Einsatzgebieten. Für Translation mit langen Verfahrwegen und eine hohe Ausnutzung der Maschine wird eine spezielle zweidimensionale Verzahnung von Rotor und Stator benötigt. Es wurden verschiedene Verzahnungstypen mit gleicher Nutteilung in Stator und Rotor untersucht, die sich in die zwei Grundtypen Schachbrettverzahnung und Kreuzverzahnung einteilen lassen. Für diese erfolgten eine Analyse und ein Vergleich der erreichbaren Kraftdichten für einen magnetischen Pol der Maschine. Die Schachbrettverzahnung erreicht dabei eine deutlich höhere Tangentialkraftdichte bei nur geringfügig niedrigerer Radialkraftdichte. Im Gegensatz zur Kreuzverzahnung sind jedoch beide Tangentialkräfte verkoppelt. Für die Ermittlung der optimalen Verzahnungsversetzungen und den Nachweis der Steuerbarkeit wurde eine Minimierung einer Zielfunktion, welche die Stromwärmeverluste im ungünstigsten Fall widerspiegelt, durchgeführt. Anhand der jeweils minimalen Zielfunktionswerte konnte gezeigt werden, dass sowohl ein schachbrettverzahnter Motor als auch ein kreuzverzahnter Motor mit sechs Polpaaren steuerbar ist. Die erreichbaren Tangentialkräfte des schachbrettverzahnten Motors liegen deutlich über jenen des kreuzverzahnten Motors, die Radialkräfte sind nur geringfügig niedriger.
\end{abstract}

Schlüsselwörter: lagerloser reluktanter Rotations-Linear-Motor; Schachbrettverzahnung; Kreuzverzahnung;

Verzahnungsversetzungen; Steuerbarkeit; Zielfunktion

\section{Two-dimensional toothing for bearingless reluctant rotary-linear motors.}

The bearingless rotary-linear reluctance motor is a novel drive and allows independent magnetic levitation as well as translation and rotation of a ferromagnetic rotor with a single compact actuator. With the simple and robust design, this motor allows a wide range of new applications. For translation with long travel lengths and high utilization of the machine a special two-dimensional toothing of rotor and stator is needed. Different toothing types with the same tooth pitch in stator and rotor were investigated. These can be divided into the two basic types of chessboard toothing and cross toothing. For this, an analysis and a comparison of the attainable force densities for one magnetic pole of the machine were carried out. The chessboard toothing attains significantly higher tangential forces at only slightly less radial force. In contrast to the cross toothing, however, both tangential forces are coupled. A minimization of a target function, which reflects the heat losses in the worst case, was carried out to determine the optimal toothing offsets and to prove controllability. Based on the respective minimum target function values, it was shown that both a chessboard toothed motor and a cross toothed motor with each six pole pairs are controllable. The attainable tangential forces of the chess field toothed motor are significantly higher than those of the cross toothed motor. The radial forces are only slightly lower.

Keywords: bearingless rotary-linear reluctance motor; chessboard toothing; cross toothing; toothing offsets; controllability; target fuction

Eingegangen am 11. Oktober 2017, angenommen am 18. Jänner 2018, online publiziert am 28. März 2018 (C) The Author(s) 2018

\section{Einleitung}

Aktive Magnetlager bieten gegenüber herkömmlichen Lagern eine Reihe von Vorteilen, etwa Festkörperreibungs- und Verschleißfreiheit, hohe Präzision sowie Beeinflussbarkeit von Solllage und Lagerdynamik während des Betriebs [1]. Lagerlose Motoren vereinen dabei Motor und Magnetlager zu einer kompakten Einheit [2].

Konventionelle reluktante Rotations-Linear-Motoren bestehen aus einem rotierenden Motor und einem Linear-Motor, welche mechanisch miteinander verbunden sind und besitzen mechanische Lager für den Rotor. In [3] wird ein rotierender Motor um zwei weitere Statoren ergänzt, die axiale Hübe ermöglichen. Da der mittlere Teil des Rotors den zugehörigen Rotations-Stator nicht verlassen darf, ist der Verfahrweg auf kleine Werte begrenzt. Der Rotations-Linear-
Motor in [4] besteht aus einem Rotations-Stator und einem dreiteiligen Linear-Stator. Der Rotor ist zweidimensional verzahnt, indem sowohl durchgängige Längsnuten als auch durchgängige Quernuten eingebracht sind. Dadurch ist der axiale Verfahrweg nur durch die Rotorlänge begrenzt. Beide genannten Motorkonzepte benötigen mechanische Lagerungen und sind verhältnismäßig aufwändig zu fertigen.

Schleicher, André, Professur elektrische Energiewandlungssysteme und Antriebe, Technische Universität Chemnitz, Reichenhainer Straße 70, 09126 Chemnitz, Deutschland (E-Mail: andre.schleicher@etit.tu-chemnitz.de); Werner, Ralf, Professur elektrische Energiewandlungssysteme und Antriebe, Technische Universität Chemnitz, Reichenhainer Straße 70, 09126 Chemnitz, Deutschland 


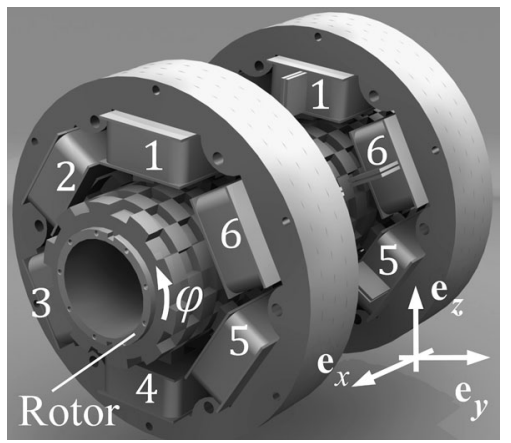

Abb. 1. CAD-Modell des lagerlosen reluktanten Rotations-Linear-Motors

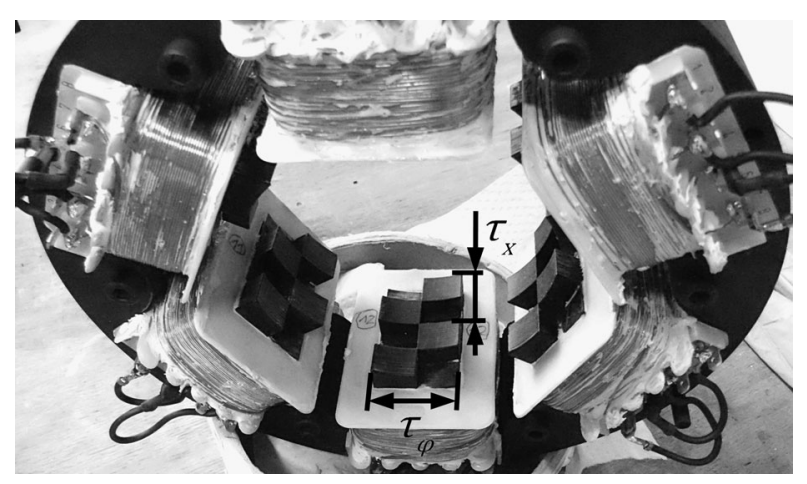

Abb. 2. Stator mit sechs schachbrettverzahnten Polen während der Fertigung

Der in diesem Beitrag betrachtete neuartige lagerlose reluktante Rotations-Linearmotor benötigt keine mechanischen Lager und besteht aus zwei gleichartigen Statoren. Daher ist dieser Antrieb sehr kompakt, robust sowie einfach zu fertigen und ermöglicht vielfältige Einsatzgebiete. Besonders geeignet ist der Motor für komplexe Bewegungsabläufe sowie für Positionier- und Transportanwendungen im Vakuum oder in Reinräumen. Für die Vorschubkraft und das Drehmoment wird jedoch eine zweidimensionale Verzahnung in Stator und Rotor benötigt. Schwerpunkt der Betrachtungen in diesem Beitrag ist die Entwicklung von zweidimensionalen Verzahnungen mit gleicher Nutteilung in Stator und Rotor sowie die Untersuchung der Steuerbarkeit und der erreichbaren Kraftdichten. Aufgrund der magnetischen Lagerung des Rotors spielt dabei im Gegensatz zu konventionellen Rotations-Linear-Motoren auch die radiale Kraftdichte eine Rolle.

\section{Grundaufbau lagerloser reluktanter \\ Rotations-Linear-Motor}

Der in diesem Beitrag betrachtete lagerlose Rotations-Linear-Motor besteht aus zwei gleichartigen Statoren mit jeweils sechs Polspulenwicklungen, siehe Abb. 1. Die zweidimensionale Verzahnung besitzt in Stator und Rotor die gleichen Nutteilungen $\tau_{x}$ und $\tau_{\varphi}$, welche wiederum beide in etwa gleich groß sind, siehe Abb. 2. Jeweils zwei gegenüberliegende Pole eines Statorteils (z. B. 1 und 4 in Abb. 1) sind gleichartig verzahnt, wodurch eine Trennbarkeit von radialer Kraft und Vorschubkraft erreicht werden kann. Die Verzahnungen der verschiedenen Polpaare wiederum weisen unterschiedlichen Versatz zur Rotorverzahnung auf.

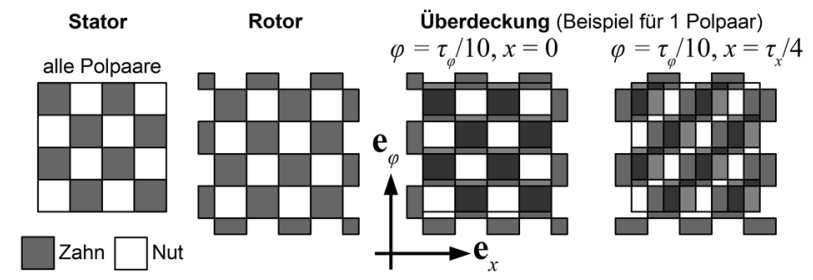

Abb. 3. Schachbrettverzahnung

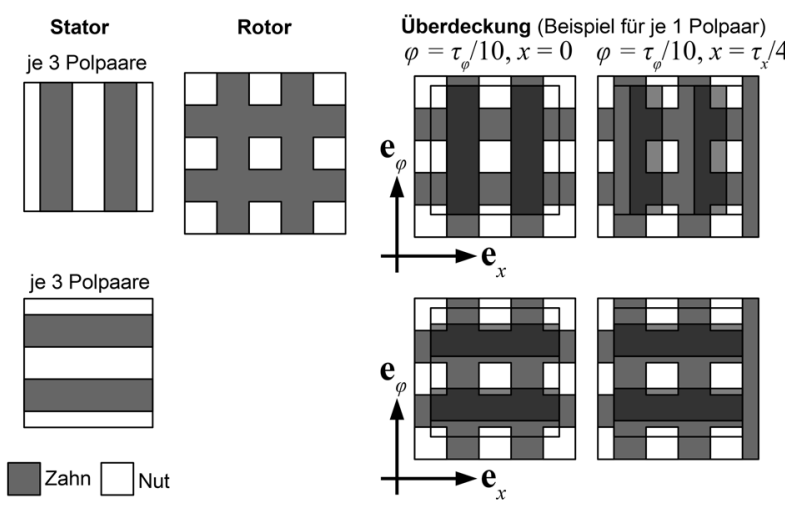

Abb. 4. Kreuzverzahnung

\section{Grundtypen der Verzahnungen}

Im Folgenden werden die Verzahnungen der gekrümmten Poloberflächen als Muster in der zweidimensionalen $x \varphi$-Ebene (vgl. Abb. 1) betrachtet, die durch die Einheitsvektoren $\mathbf{e}_{x}$ und $\mathbf{e}_{\varphi}$ aufgespannt wird. Die gefundenen Verzahnungen, welche für lagerlose reluktante Rotations-Linear-Motoren geeignet sind, lassen sich in zwei Grundtypen einteilen. Dargestellt sind in den folgenden Abbildungen von links nach rechts die Verzahnung eines Pols des Stators, die Verzahnung des Rotors sowie die Überdeckung beider Verzahnungen. Letztere veranschaulicht die Relativposition von Stator- und Rotorzähnen für zwei verschiedene Rotorpositionen $x$.

\section{Schachbrettverzahnung}

Gemäß Abb. 3 sind der Rotor und alle Pole des Stators mit Zähnen und Nuten rechteckiger Oberfläche versehen. Diese wechseln sich wie bei einem Schachbrett in beiden Koordinatenrichtungen $a b$. Für die Kraftwirkung entscheidend ist die Änderungsrate des magnetischen Widerstands bei Verschiebung des Rotors in radiale Richtung oder in $x$ - bzw. $\varphi$-Richtung. Diese wiederum hängt maßgeblich von der Überdeckungsfläche der Zähne von Rotor und Stator sowie deren Änderungsrate bei Verschiebung ab. Bei der Schachbrettverzahnung sind daher beide Vorschubkräfte (axiale Kraft $F_{X}$ und Umfangskraft $F_{\varphi}$ ) im Allgemeinen miteinander verkoppelt, da sich die Überdeckungsfläche eines Pols mit dem Rotor sowohl bei Verschiebung in $x$ - als auch in $\varphi$-Richtung verändert (vgl. Überdeckung in Abb. 3):

2. Kreuzverzahnung

Drei Polpaare des Stators sind längsverzahnt, die anderen drei sind querverzahnt. Der Rotor ist durchgängig kreuzartig verzahnt. Im Gegensatz zur Schachbrettverzahnung gibt es dadurch eine Entkopplung der Kräfte. So ändert sich die Überdeckungsfläche in Abb. 4 oben rechts nur bei Verschiebung in $x$-Richtung, während sich die Überdeckungsfläche der anderen drei Polpaare (Abb. 4 rechts unten) nur bei Verschiebung in $\varphi$-Richtung ändert. Somit würden bei einem Motor mit sechs Polpaaren drei Polpaare nur eine axiale Vorschubkraft erzeugen, die nur von der 


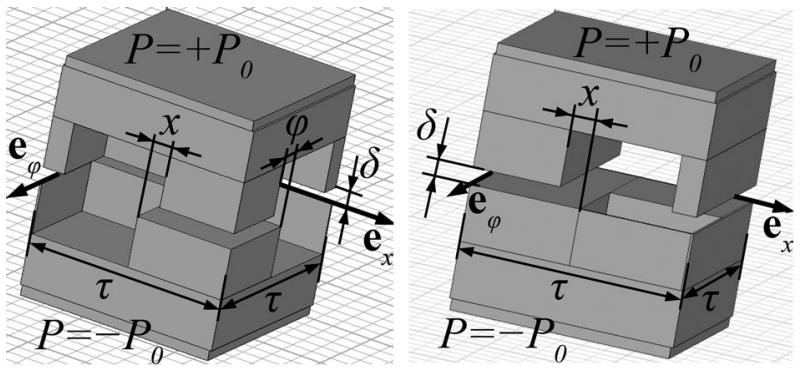

Abb. 5. FEM-Modelle, links Schachbrettverzahnung, rechts Kreuzverzahnung

Verschiebung $x$ abhängt, während die anderen drei nur eine Umfangskraft erzeugen, welche nur vom Winkel $\phi$ abhängt. Von der Kreuzverzahnung sind Untervarianten mit gleicher Statorverzahnung, jedoch anderer Rotorverzahnung denkbar. Beispiele wären eine treppenförmige Verzahnung oder eine invertierte Kreuzverzahnung, bei der Zähne und Nuten vertauscht sind, wie diese etwa in [4] eingesetzt wird. Letztere besitzt jedoch aufgrund der deutlich geringeren mittleren Überdeckungsfläche nur niedrige mittlere radiale Kräfte und ist somit für den lagerlosen Motor ungeeignet.

In den folgenden Betrachtungen wird zunächst für beide Verzahnungsarten die optimale Geometrie eines verzahnten Pols bestimmt. Anschließend werden die optimalen Versetzungen der Verzahnungen ermittelt und die Steuerbarkeit eines Motors mit der jeweiligen Verzahnungsart überprüft. Abschließend werden die erreichbaren Kräfte von je einem vollständigen Motor mit Schachbrett- und Kreuzverzahnung miteinander verglichen.

\section{Ermittlung der Kräfte eines Pols für beide Verzahnungstypen}

\subsection{Numerische Berechnung der Kräfte mittels FEM}

Zur Bestimmung der Kräfte eines schachbrettverzahnten oder kreuzverzahnten Pols auf den Rotor wurde die Laplace-Gleichung für das magnetische Potential $P$ mittels FEM gelöst. Daraus erfolgte die Ermittlung des magnetischen Felds $\mathbf{H}$, der Flussdichte $\mathbf{B}$ und schließlich über die Änderung der magnetischen Energie $W_{\text {mag }}$ die magnetische Kraft $\mathbf{F}$ :

$$
\nabla^{2} P=0, \quad \mathbf{H}=-\nabla P, \quad \mathbf{B}=\mu \mathbf{H}, \quad \mathbf{F}=-\nabla W_{\text {mag }}
$$

Darin ist $\mu$ die Permeabilität, welche für Rotor und Stator als ideal $(\mu \rightarrow \infty)$ angesetzt ist. Es erfolgt für beide Verzahnungen jeweils nur die Berechnung der kleinsten periodische Zelle, siehe Abb. 5 . Die Nutteilung ist für beide Richtungen gleich: $\tau_{x}=\tau_{\varphi}=\tau$.

Die Mantelflächen der Verzahnungsmodelle besitzen periodische Randbedingungen, während Boden- und Deckfläche jeweils mit einem konstanten Potential $+P_{0}$ bzw. $-P_{0}$ versehen sind. Durch die Berechnung einer solchen Zelle anstatt eines konkreten Motorausschnitts werden die Ergebnisse unabhängig von anderen Parametern wie Rückenhöhe, Statordurchmesser, etc. und somit besser vergleichbar und übertragbar. Ebenso wird die Rechenzeit erheblich reduziert. Mitunter werden für Reluktanzmotoren unverzahnte Pole genutzt. In diesem Fall können der Pol als mit nur einem Zahn verzahnt und die o.g. FEM-Modelle als Näherung betrachtet werden.

Die Beträge der berechneten Kräfte sind aufgrund der Linearität des Problems skalierbar über folgende Relationen:

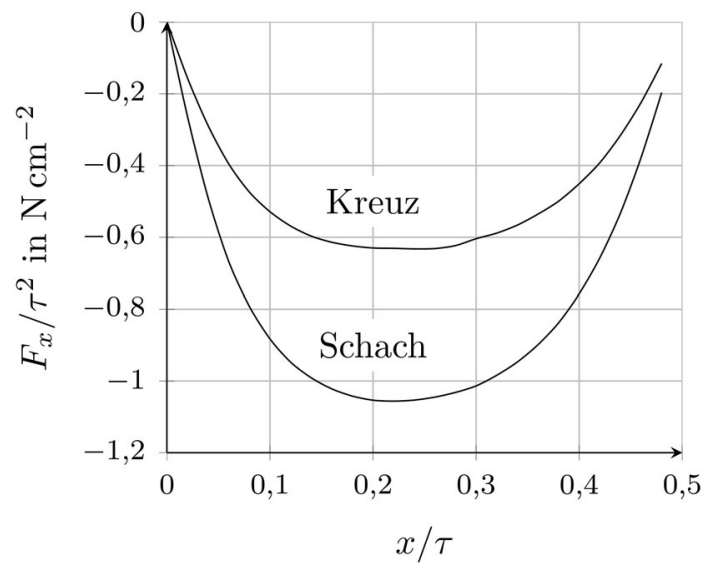

Abb. 6. Tangentialkraftdichte in Abhängigkeit der Verzahnungsverschiebung für $\frac{\tau}{\delta}=13$

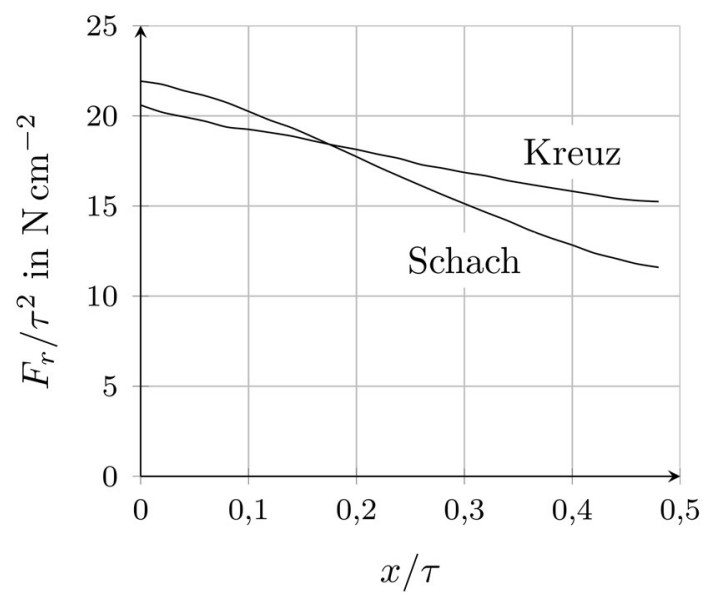

Abb. 7. Radialkraftdichte in Abhängigkeit der Verzahnungsverschiebung für $\frac{\tau}{\delta}=13$

$$
F \sim \tau^{2} B^{2} \sim \tau^{2}\left(P_{0} / \delta\right)^{2}
$$

Darüber hinaus behält die Kraft ihre Richtung, wenn alle Abmessungen gleich skaliert werden, d. h. das Verhältnis $\tau / \delta$ von Nutteilung zu Luftspaltlänge konstant bleibt. Daher wurde die Rechnung für nur eine feste Luftspaltlänge von $\delta=1 \mathrm{~mm}$ durchgeführt. Das Potential $P_{0}$ wurde ebenfalls für beide Verzahnungen fest eingestellt und beträgt $P_{0}=400 \mathrm{~A}$, was zu einer mittleren Induktion von $B_{a v g} \approx 1 \mathrm{~T}$ im Luftspalt zwischen zwei gegenüberliegenden Zähnen führt. Im Interesse möglichst großer Vorschubkräfte wurde zunächst die Nutteilung $\tau$ des Modells variiert, um maximale Tangentialkraftdichte $\sigma_{x}=F_{x} / \tau^{2}$ zu erreichen. Dabei erfolgte für jede Nutteilung $\tau$ eine Variation der Verschiebung $x$ der Verzahnungen und eine Ermittlung des globalen Maximums von $\sigma_{x}$. In Abb. 6 bzw. Abb. 7 ist der Verlauf von Tangential- bzw. Radialkraftdichte exemplarisch für das Verhältnis $\tau / \delta=13$ dargestellt. Der Verlauf des Tangentialkraftmaximums ist für beide Verzahnungen in Abb. 8 dargestellt und besitzt wiederum für je ein bestimmtes Verhältnis $\tau / \delta$ ein globales Maximum. Für alle folgenden Betrachtungen wird von diesem optimalen geometrischen Verhältnis ausgegangen. Die sich einstellende Feldverteilung $\mathbf{B}$ ist in Abb. 9 jeweils als achsparalleler Schnitt durch die Mitte der Zähne (Schnittebenen-Normalform: Schachbrett: $\mathbf{r} \cdot \mathbf{e}_{\varphi}=1 / 4 \tau$, Kreuzverzahnung: $\mathbf{r} \cdot \mathbf{e}_{\varphi}=3 / 4 \tau$, vgl. Abb. 5) dargestellt. Dabei sind oberer 


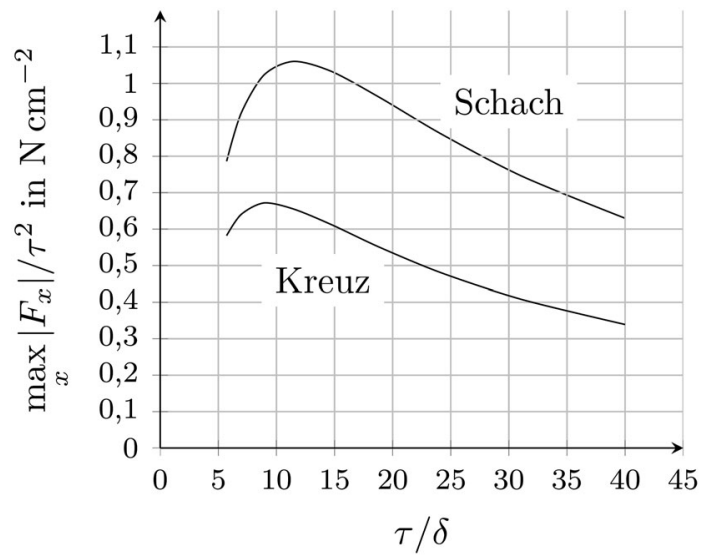

Abb. 8. Maximale Tangentialkraftdichte in Abhängigkeit der Geometrie

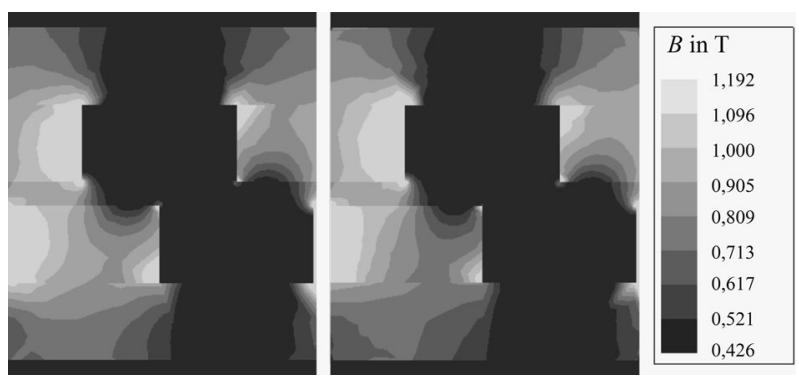

Abb. 9. Flussdichteverteilungen im Schnitt, links: Schachbrettverzahnung, rechts: Kreuzverzahnung

Tab. 1. FEM-Ergebnisse der erreichbaren Kräfte

\begin{tabular}{lll}
\hline & \multicolumn{2}{l}{ Ergebnisse FEM Berechnung für $B_{a v g} \approx 1 \mathrm{~T}$} \\
\cline { 2 - 3 } & $\begin{array}{ll}\text { max. Tangential- } \\
\text { kraftdichte }\end{array}$ & $\begin{array}{l}\text { mittlere Radial- } \\
\text { kraftdichte }\end{array}$ \\
& $\sigma_{x, \max }=\frac{\max _{x}\left|F_{x}\right|}{\tau^{2}}$ & $\sigma_{r, a v g}=\frac{\frac{1}{\tau} \int_{x=0}^{\tau} F_{r} \mathrm{~d} x}{\tau^{2}}$ \\
\hline Schachbrett & $1,06 \mathrm{~N} \mathrm{~cm}^{-2}$ & $17,4 \mathrm{~N} \mathrm{~cm}^{-2}$ \\
Kreuz & $0,67 \mathrm{~N} \mathrm{~cm}^{-2}$ & $19,1 \mathrm{~N} \mathrm{~cm}^{-2}$ \\
\hline
\end{tabular}

und unterer Modellteil um $x=\tau / 4$ und $\varphi=0$ zueinander verschoben.

\subsection{Vergleich der erreichbaren Kräfte für beide Verzahnungstypen}

Zum Vergleich beider Verzahnungen wurden bei optimalem Verhältnis $\tau / \delta$ jeweils die Kräfte beider Verzahnungsmodelle ermittelt. Die Ergebnisse der FEM-Berechnungen für beide Verzahnungstypen werden in Tab. 1 hinsichtlich ihrer erreichbaren Tangential- und Radialkraftdichten verglichen. Die erreichbare Tangentialkraftdichte ist bei Schachbrettverzahnung deutlich größer als bei Kreuzverzahnung, die mittlere Radialkraftdichte ist jedoch nur geringfügig kleiner als bei Kreuzverzahnung:

$$
\frac{\sigma_{x, \text { max }, \text { Kreuz }}}{\sigma_{x, \text { max }, \text { Schach }}}=0,63, \quad \frac{\sigma_{r, \text { avg }, \text { Kreuz }}}{\sigma_{r, \text { avg,Schach }}}=1,10
$$

Insgesamt ist anzumerken, dass diese Ergebnisse den Idealfall darstellen, da das Materialverhalten idealisiert wurde. Zudem ist die Induktionsverteilung in den Zähnen von der Verzahnungsart und den Verschiebungen $x$ bzw. $\varphi$ abhängig. Der Unterschied der mittleren Zahninduktion ist zwischen beiden Verzahnungsarten jedoch nur gering, was u.a. in der Schnittebene in Abb. 9 ersichtlich ist. Daher ist die Vergleichbarkeit der Verzahnungsarten mittels der Modelle gewährleistet. Wäre die Induktionsverteilung sehr unterschiedlich, gäbe es aufgrund von Sättigungseffekten in der Praxis größere Abweichungen von den Verhältnissen aus (3).

\subsection{Abhängigkeiten der tangentialen Kräfte eines Polpaars von der Rotorverschiebung}

Die axiale Vorschubkraft bzw. das Drehmoment eines Pols des Motors kann mittels der Kraftdichte $\sigma$ aus dem vorherigen Abschnitt berechnet werden. Wegen Relation (2) hängt diese Kraft vom magnetischen Fluss $\phi$ durch den Pol ab:

$$
F_{x, P o l}=\sigma_{x} A \sim B^{2} A \sim \frac{\phi^{2}}{A}
$$

Darin ist $A$ die Fläche des Pols. Die Kraft des $j$-ten Polpaars ergibt sich aus der Summe der Kräfte beider gegenüberliegender Pole:

$$
F_{x, j} \sim \frac{\Phi_{j}}{A_{j}}
$$

Darin ist $\Phi_{j}$ die Summe der Quadrate der beiden Flüsse durch die Pole und ein Element des Flussquadratevektors $\boldsymbol{\Phi}$, der wie folgt definiert ist:

$$
\boldsymbol{\Phi}=\left(\begin{array}{c}
\Phi_{1} \\
\vdots \\
\Phi_{6}
\end{array}\right):=\left(\begin{array}{c}
\phi_{1 A}^{2}+\phi_{4 A}^{2} \\
\phi_{2 A}^{2}+\phi_{5 A}^{2} \\
\phi_{3 A}^{2}+\phi_{6 A}^{2} \\
\phi_{1 B}^{2}+\phi_{4 B}^{2} \\
\phi_{2 B}^{2}+\phi_{5 B}^{2} \\
\phi_{3 B}^{2}+\phi_{6 B}^{2}
\end{array}\right)
$$

Die Indizes A und B bezeichnen den vorderen bzw. hinteren Statorteil mit jeweils sechs Polen, vgl. Abb. 1. So ist beispielsweise $\Phi_{3}=\phi_{3 A}^{2}+\phi_{6 A}^{2}$ verantwortlich für die Kraft des Polpaars 3 bestehend aus Pol 3 und Pol 6 des vorderen Statorteils und setzt sich aus den Flüssen $\phi_{3 A}$ sowie $\phi_{6 A}$ durch diese beiden Pole zusammen.

Bei der Kreuzverzahnung hängt die Tangentialkraftdichte nur von der ihr zugehörigen Rotor-Verschiebungskoordinate $x$ bzw. $\varphi$ ab. Somit können die Kräfte des Pols wie folgt in ein Fourierpolynom 1. Grades entwickelt werden:

$$
\begin{aligned}
& F_{X, \text { Kreuz }, j}(\tilde{x}) \approx-C_{\text {Kreuz }} \sin \left(\tilde{x}+\Delta \tilde{x}_{j}\right) \cdot \Phi_{j} \\
& F_{\varphi, \text { Kreuz }, j}(\tilde{\varphi}) \approx-C_{\text {Kreuz }} \sin \left(\tilde{\varphi}+\Delta \tilde{\varphi}_{j}\right) \cdot \Phi_{j}
\end{aligned}
$$

Darin sind $\tilde{x}$ und $\tilde{\varphi}$ zur Vereinfachung der Schreibweise normierte Koordinaten:

$$
\tilde{x}:=\frac{2 \pi}{\tau_{x}} x, \quad \tilde{\varphi}:=\frac{2 \pi}{\tau_{\varphi}} \varphi
$$

$\Delta \tilde{x}_{j}$ und $\Delta \tilde{\varphi}_{j}$ sind die relativen Versetzungen der Verzahnung des $j$ ten Polpaars gegenüber einem anderen festgelegten Bezugspolpaar, vgl. Abb. 10 und 11.

Die entsprechende Fourier-Näherung für das $j$-te Polpaar mit Schachbrettverzahnung lautet:

$$
\begin{aligned}
& F_{x, \operatorname{Schach}, j}(\tilde{x}, \tilde{\varphi}) \approx-C_{\text {Schach }} \sin \left(\tilde{x}+\Delta \tilde{x}_{j}\right) \cdot \cos \left(\tilde{\varphi}+\Delta \tilde{\varphi}_{j}\right) \cdot \Phi_{j} \\
& F_{\varphi, \text { Schach }, j}(\tilde{x}, \tilde{\varphi}) \approx-C_{\text {Schach }} \cos \left(\tilde{x}+\Delta \tilde{x}_{j}\right) \cdot \sin \left(\tilde{\varphi}+\Delta \tilde{\varphi}_{j}\right) \cdot \Phi_{j}
\end{aligned}
$$

In Abb. 12 ist exemplarisch der Verlauf der mittels FEM berechneten axialen Kraft $F_{x}$ eines Polpaars in Abhängigkeit der Verschiebung $\tilde{x}$ für Kreuzverzahnung dargestellt, sowie die analytische Näherung nach Gl. (7) mit minimalem quadratischen Fehler durch optimale Wahl von $C_{\text {Kreuz }}$. Abb. 13 zeigt die Kraft $F_{X}$ in Abhängigkeit der Ver- 

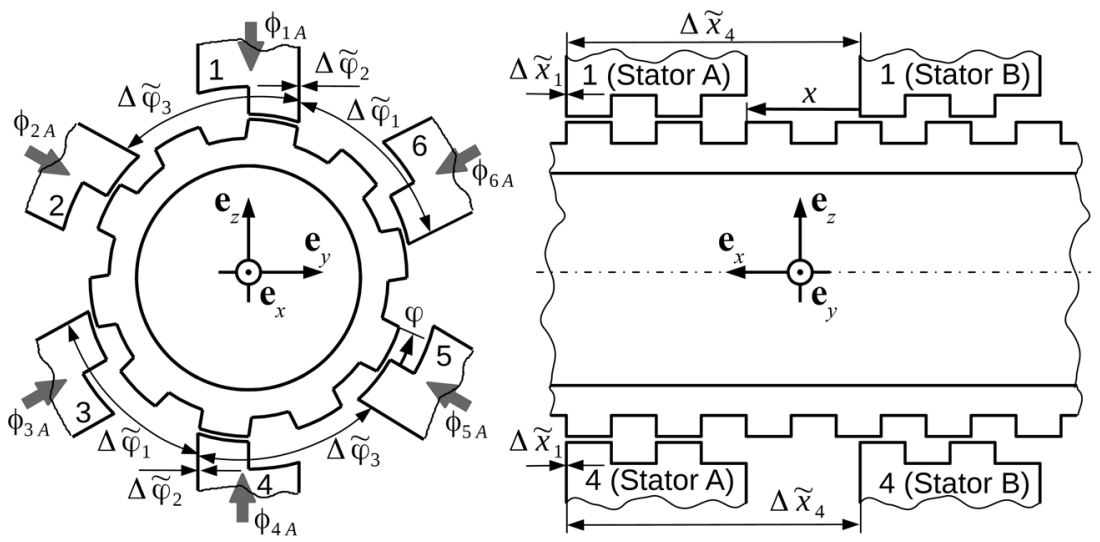

Abb. 10. Schnitte durch optimal schachbrettverzahnten Motor
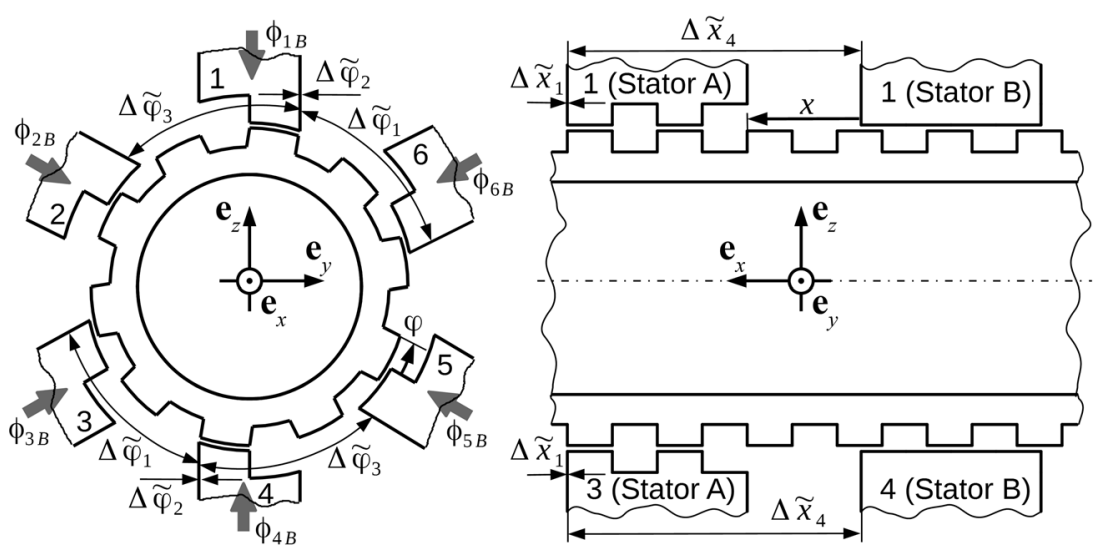

Abb. 11. Schnitte durch optimal kreuzverzahnten Motor

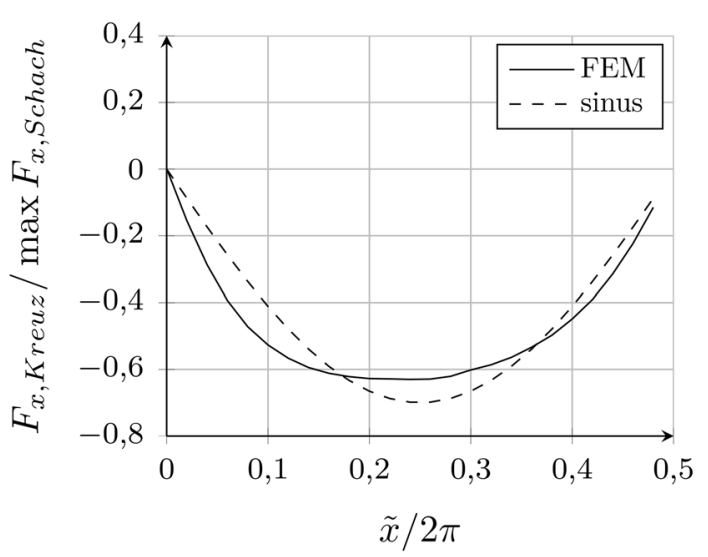

Abb. 12. Tangentialkraftverlauf bei optimaler Kreuzverzahnung

schiebungen $\tilde{x}$ und $\tilde{\varphi}$ für die Schachbrettverzahnung sowie die Nä-

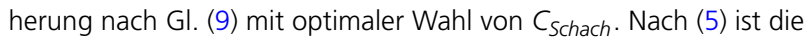
Fläche $A$ der der Pole entscheidend für den Absolutwert der Kräfte. Da $A$ jedoch für beide Verzahnungen gleich sein soll, sind beide Kraftverläufe auf die maximale Kraft des schachverzahnten Polpaars normiert. In Übereinstimmung mit (3) gilt dabei für die Fourierkoeffizienten:

$$
C_{\text {Kreuz }} \approx 0,63 C_{\text {Schach }}
$$

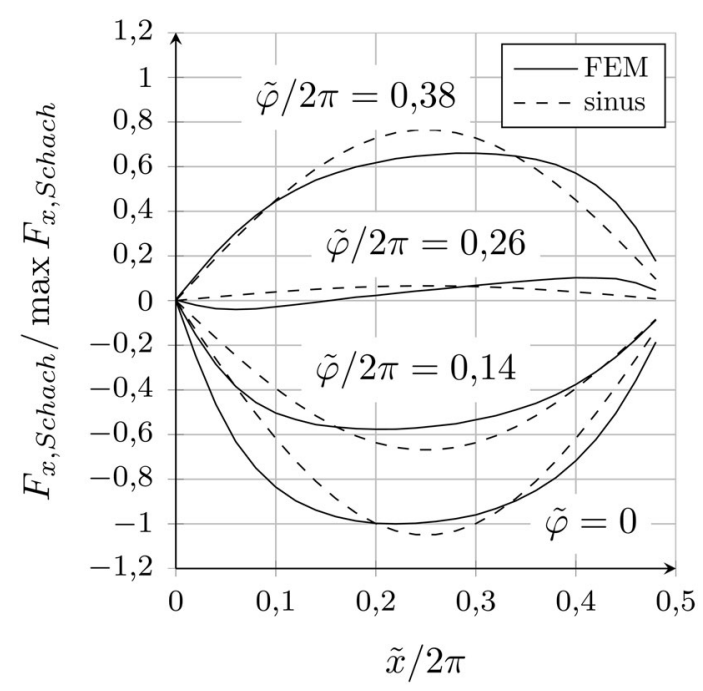

Abb. 13. Tangentialkraftverlauf bei optimaler Schachbrettverzahnung

Zwar sind in beiden Diagrammen Abweichungen der FEM-Ergebnisse von der Sinusform erkennbar, jedoch würden in der Praxis die Zahnsättigung und der Einfluss der Kantenbearbeitung des Materials den tatsächlichen Oberwellengehalt reduzieren. Fortan werden 
die Sinus-Näherungen verwendet, um zu vermeiden, dass bei der in den nächsten Abschnitten beschriebenen Optimierung unerwünschte lokale Nebenoptima durch die Oberwellen entstehen. Zudem wird dadurch die Rechenzeit deutlich reduziert. Mit Hilfe der Tangentialkraftverläufe $F_{X, j}$ und $F_{\varphi, j}$ eines Polpaars nach (7) bzw. (9) lassen sich die resultierenden Vorschubkräfte $\mathbf{f}$ des gesamten Motors mit sechs Polpaaren berechnen:

$$
\mathbf{f}:=\left(\begin{array}{c}
F_{X} \\
F_{\varphi}
\end{array}\right)=\sum_{j=1}^{6}\left(\begin{array}{c}
F_{X, j} \\
F_{\varphi, j}
\end{array}\right)=\left(\begin{array}{lll}
G_{11} & \cdots & G_{16} \\
G_{21} & \cdots & G_{26}
\end{array}\right)\left(\begin{array}{c}
\Phi_{1} \\
\vdots \\
\Phi_{6}
\end{array}\right)=\mathbf{G} \boldsymbol{\Phi}
$$

Die Matrix G beinhaltet die entsprechenden Sinusterme aus (7) bzw. (9), sodass diese von der tangentialen Verschiebung des Rotors $\mathbf{r}$ sowie den individuellen Versetzungen $\mathbf{p}$ der Verzahnungen der Polpaare zur Rotorverzahnung (vgl. Abb. 10 und 11) abhängt:

$$
\mathbf{G}=\mathbf{G}(\mathbf{r}, \mathbf{p}), \quad \operatorname{mit} \mathbf{r}:=\left(\begin{array}{c}
\tilde{x} \\
\tilde{\varphi}
\end{array}\right), \mathbf{p}:=\left(\begin{array}{ccc}
\Delta \tilde{x}_{1} & \cdots & \Delta \tilde{x}_{6} \\
\Delta \tilde{\varphi}_{1} & \cdots & \Delta \tilde{\varphi}_{6}
\end{array}\right)
$$

Für Kreuz- bzw. Schachbrettverzahnung lautet $\mathbf{G}$ wie folgt:

$$
\begin{gathered}
\mathbf{G}_{\text {Kreuz }}(\mathbf{r}, \mathbf{p})=-C_{\text {kreuz }}\left(\begin{array}{lll}
\sin \left(x+\Delta \tilde{x}_{1}\right) & \cdots & \sin \left(x+\Delta \tilde{x}_{6}\right) \\
\sin \left(\tilde{x}+\Delta \tilde{x}_{1}\right) & \cdots & \sin \left(\tilde{x}+\Delta \tilde{x}_{6}\right)
\end{array}\right) \\
\mathbf{G}_{\text {schach }}(\mathbf{r}, \mathbf{p})=-C_{\text {schach }}\left(\begin{array}{ll}
\sin \left(x+\Delta \tilde{x}_{1}\right) \cos \left(\tilde{\varphi}+\Delta \tilde{\varphi}_{1}\right) \\
\cos \left(\tilde{x}+\Delta \tilde{x}_{1}\right) \sin \left(\tilde{\varphi}+\Delta \tilde{\varphi}_{1}\right) \\
\cdots & \sin \left(x+\Delta \tilde{x}_{6}\right) \cos \left(\tilde{\varphi}+\Delta \tilde{\varphi}_{6}\right) \\
\cdots & \cos \left(\tilde{x}+\Delta \tilde{x}_{6}\right) \sin \left(\tilde{\varphi}+\Delta \tilde{\varphi}_{6}\right)
\end{array}\right)
\end{gathered}
$$

Um Steuerbarkeit des Motors zu gewährleisten, müssen alle Verzahnungsversetzungen in $\mathbf{p}$ so gewählt werden, dass mit dem Motor in jeder beliebigen Position $\mathbf{r}$ des Rotors Vorschubkräfte $\mathbf{f}$ mit beliebiger Richtung erzeugt werden können.

\section{Optimierung der Verzahnungsversetzungen}

\subsection{Zielfunktion}

Die Steuerbarkeit des Motors, welche im vorherigen Abschnitt erläutert wurde, bedeutet dass das Gleichungssystem (11) stets nach $\Phi$ auflösbar ist:

$$
\exists \boldsymbol{\Phi}(\mathbf{f}, \mathbf{r}, \mathbf{p}) \quad \text { mit } \mathbf{f}=\mathbf{G}(\mathbf{r}, \mathbf{p}) \cdot \boldsymbol{\Phi} \forall \mathbf{r}, \mathbf{f}
$$

Dies impliziert erstens vollen Rang von $\mathbf{G}$ und zweitens dürfen alle Einträge des Flussquadratevektors $\boldsymbol{\Phi}$ nach dessen Definition (6) nur positive Größen sein:

$$
\Phi_{j} \geq 0 \forall j
$$

Die Flussquadrate korrelieren mit den Wicklungsströmen des Motors. Um die Stromwärmeverluste zu reduzieren, soll daher die Norm des Flussquadratevektors $\boldsymbol{\Phi}$ minimiert werden:

$$
\|\boldsymbol{\Phi}(\mathbf{f}, \mathbf{r}, \mathbf{p})\| \rightarrow \min
$$

Diese Minimierung erfolgt praktisch über die Wahl der Wicklungsströme durch die Motorsteuerung und in der Berechnung über einen Optimierungsalgorithmus (quadratische Programmierung).

Für verschiedene Kräfte $\mathbf{f}$ ergeben sich nun bei steuerbarem Motor verschiedene solcher optimalen Lösungen $\boldsymbol{\Phi}$. Größere Kräfte können stets durch einfache Skalierung von $\boldsymbol{\Phi}$ erreicht werden, nach (11) gilt $\|\mathbf{f}\| \sim\|\boldsymbol{\Phi}\|$. Daher ist nur die Richtung der Kraft entscheidend, sodass nun $\mathbf{f}$ wie folgt gewählt wird:

$$
\mathbf{f}=\mathbf{f}(\alpha)=\left(\begin{array}{c}
\cos \alpha \\
\sin \alpha
\end{array}\right) \quad \text { mit } \alpha \in[0,2 \pi)
$$

Nun wird eine Zielfunktion $z$ in Abhängigkeit der Versetzungen $\mathbf{p}$ definiert, welche ein $M a ß$ für die Stromwärmeverluste bei optimaler Ansteuerung, jedoch für den ungünstigsten Fall aus aufzubringender Kraftrichtung $\mathbf{f}$ und Rotorposition $\mathbf{r}$ ist:

$$
z(\mathbf{p}):=\max _{\mathbf{r}, \alpha} \min \|\boldsymbol{\Phi}(\mathbf{f}(\alpha), \mathbf{r}, \mathbf{p})\|
$$

Diese Zielfunktion $z$ gilt es nun durch geeignete Wahl der Versetzungsmatrix $\mathbf{p}$ zu minimieren. Ist $z$ nicht existent oder divergent, so ist der Motor nicht steuerbar, d. h. eine bestimmte Kraft kann nicht erzeugt werden oder an mindestens einer Rotorposition $\mathbf{r}$ können die gewünschten Kräfte nicht erzeugt werden.

Als Nebenbedingungen für die Optimierung von z gelten GI. (11) und Ungl. (16). Weiterhin sollen die Kräfte eine negative Ableitung in ihre zugehörige Koordinatenrichtung aufweisen:

$$
\frac{\partial F_{X}}{\partial \tilde{x}}=\sum_{j=1}^{6} \frac{\partial G_{1 j}}{\partial \tilde{x}} \Phi_{j}<0, \quad \frac{\partial F_{\varphi}}{\partial \tilde{\varphi}}=\sum_{j=1}^{6} \frac{\partial G_{2 j}}{\partial \tilde{\varphi}} \Phi_{j}<0
$$

Diese zusätzliche Nebenbedingung wirkt sich günstig auf die Stabilität aus, da der Rotor bei kleinen Auslenkungen aus der Ruheposition r eine rücktreibende Kraft erfahren würde, was eine Lageregelung des Antriebs erleichtert. Positive Ableitungen hingegen äußern sich als "negative Steifigkeiten" und müssten durch eine Regelung mit entsprechend größerer Reglerverstärkerung ausgeglichen werden.

Die Optimierung der Zielfunktion $z$ ist trivial wenn Kreuzverzahnung eingesetzt wird. Durch die Trennung beider Koordinatenachsen lässt sich diese auf zwei getrennte Optimierungen zurückführen, worauf im nächsten Abschnitt eingegangen wird.

Für die Schachbrettverzahnung hingegen ist die Optimierung nichttrivial aufgrund der Verkopplungen und der Nichtlinearität der Funktionen in den Einträgen in Matrix G, vgl. (11) und (14). Daher ist zunächst weder bekannt, welcher Zielfunktionswert $z$ bei Schachbrettverzahnung im Vergleich zu Kreuzverzahnung erreichbar ist, noch ob für nur sechs unabhängige Polpaare bereits eine Lösung existiert oder mehr Polpaare benötigt werden. Daher wurde für die Schachbrettverzahnung ein globaler numerischer Optimierungsalgorithmus für die Suche der optimalen Versetzungen verwendet. Minimum und Maximum aus (19) wurden dazu ebenfalls numerisch berechnet.

\subsection{Ergebnisse Kreuzverzahnung}

Im Falle der Kreuzverzahnung kann das Problem als Optimierung zweier unabhängiger Motoren betrachtet werden. Es müssen jene drei Polpaare, welche die axiale Vorschubkraft $F_{x}$ erzeugen, optimal angeordnet werden sowie jene drei Polpaare, welche das Drehmoment $M_{\varphi}$ erzeugen. Die optimalen Versetzungen müssen dazu aus Symmetriegründen je ein Drittel der Nutteilung betragen, was bereits von konventionellen dreiphasigen Reluktanzmotoren mit gleicher Nutteilung in Stator und Rotor bekannt ist. Die zugehörige optimale Versetzungsmatrix $\mathbf{p}_{\text {Kreuz }}$ lautet damit:

$$
\mathbf{p}_{\text {Kreuz }}=\left(\begin{array}{cccccc}
-\frac{2 \pi}{3} & 0 & \frac{2 \pi}{3} & \cdot & \cdot & \cdot \\
\cdot & \cdot & \cdot & -\frac{2 \pi}{3} & 0 & \frac{2 \pi}{3}
\end{array}\right)
$$

In Abb. 11 sind Schnitte durch einen Motor mit dieser Konfiguration dargestellt. Die mit - gekennzeichneten Einträge in (21) sind frei wählbar und haben keinen Einfluss auf das Ergebnis. Zudem können die Spalten der Versetzungsmatrix ohne Beschränkung der Allgemeinheit paarweise vertauscht werden, was einer Vertauschung der Verzahnungen zweier Polpaare entspricht. Ebenso könnte ein beliebiger Wert zu allen Elementen einer Zeile addiert werden, da dies den gleichen Effekt hätte, wie eine Verschiebung des kompletten Rotors. Dies sind zwei Möglichkeiten die Fertigung der Verzahnung 
des Motors anzupassen, ohne dessen Steuerbarkeit oder Ausnutzung zu beeinträchtigen. Der zu $\mathbf{p}_{\text {Kreuz }}$ gehörige minimale Zielfunktionswert lautet:

$$
z_{\text {Kreuz }}=\frac{\sqrt{2}}{C_{\text {kreuz }}}
$$

\subsection{Ergebnisse Schachbrettverzahnung}

Der Optimierungsalgorithmus liefert für die Schachbrettverzahnung folgende Versetzungen:

$$
\mathbf{p}_{\text {Schach }}=\left(\begin{array}{cccccc}
0 & 0 & 0 & \frac{2 \pi}{4} & \frac{2 \pi}{4} & \frac{2 \pi}{4} \\
-\frac{2 \pi}{3} & 0 & \frac{2 \pi}{3} & -\frac{2 \pi}{3} & 0 & \frac{2 \pi}{3}
\end{array}\right)
$$

Diese Konfiguration entspricht einem Aufbau aus zwei gleichartigen Statoren, bei denen die Schachbrettverzahnungen der Pole je ein Drittel der Nutteilung zueinander versetzt (verdreht) sind, siehe Abb. 10. Axial gesehen besitzt die Schachbrettverzahnung des hinteren Stators (B) gegenüber der des vorderen Stators (A) einen Versatz von einer viertel Nutteilung.

Auch hier können alle Elemente einer Zeile von $\mathbf{p}_{\text {Schach }}$ um einen beliebigen Wert verschoben werden und/oder die Reihenfolge der Spalten verändert werden. Zusätzlich kann jedoch auch zu einer einzelnen Spalte ein ganzzahliges Vielfaches von $\pi$ addiert werden. Dies ergibt sich unmittelbar bei Betrachtung des Schachbrettmusters in Abb. 3 und entspricht einer Versetzung der Verzahnung um je eine halbe Nutteilung horizontal und vertikal. Der minimale Zielfunktionswert lautet:

$$
z_{\text {Schach }}=\frac{\sqrt{2}}{C_{\text {Schach }}}
$$

Aufgrund des endlichen Wertes von $z_{\text {schach }}$ ist auch der schachbrettverzahnte Motor steuerbar. Ein experimenteller Funktionsnachweis eines solchen Motors mittels Versuchsaufbau (siehe u.a. Abb. 2) wurde bereits erfolgreich auf der Tagung [5] erbracht. In späteren Veröffentlichungen soll auf die messtechnische Untersuchung der Verzahnung im Detail eingegangen werden.

\section{Vergleich der erreichbaren Kräfte eines vollständigen Motors}

Die Zielfunktionswerte sind ein Maß für die maximal zu erwartenden Stromwärmeverluste bzw. die auftretenden Flüsse durch die Pole des Motors bei Aufbringen einer Kraft des Betrags 1 mit beliebiger Richtung. Daher sind deren Kehrwerte $z^{-1}$ ein Maß für die verfügbaren Vorschubkräfte bei einer bestimmten maximalen thermischen Belastung des Motors. Ein Vergleich der jeweils minimalen Zielfunktionswerte beider Verzahnungsarten liefert:

$$
\frac{z_{\text {Kreuz }}^{-1}}{z_{\text {Schach }}^{-1}}=\frac{C_{\text {Kreuz }}}{C_{\text {Schach }}} \approx 0,63
$$

Somit liegen die erreichbaren Vorschubkräfte eines kreuzverzahnten Motors nur bei etwa $63 \%$ derer eines schachbrettartig verzahnten Motors unter sonst gleichen Bedingungen.

Die erreichbaren Radialkraftdichten hingegen sind bei Kreuzverzahnung nur ca. 10 \% höher als bei Schachbrettverzahnung, vgl. Tab. 1. Da für einen Vergleich die Polflächen beider Motoren gleichgroß sein sollen, sind die erreichbaren Radialkräfte des kreuzverzahnten Motors ebenfalls nur ca. $10 \%$ höher als die des schachbrettverzahnten Motors.

\section{Fazit und Ausblick}

Der neuartige lagerlose reluktante Rotations-Linear-Motor ermöglicht gegenüber konventionellen Antrieben eine große Vielfalt von
Bewegungen und bietet dabei die Vorteile eines magnetisch gelagerten sowie kompakten und robusten Antriebs. Für dessen Realisierung wurden zweidimensionale Verzahnungen mit gleicher Nutteilung in Stator und Rotor entwickelt, die sich in Schachbrettverzahnung und Kreuzverzahnung einteilen lassen. Mittels FEM-gestützten Berechnungen wurden beide Verzahnungen mit quaderförmigen Zähnen und jeweils optimaler Geometrie verglichen. Die Schachbrettverzahnung erreichte dabei eine deutlich höhere Tangentialkraftdichte bei nur geringfügig niedrigerer Radialkraftdichte. Die Versetzungen der Verzahnungen der Statorpole eines lagerlosen reluktanten Rotations-Linear-Motors zur Rotorverzahnung sind entscheidend für die Erzeugung der tangentialen Gesamtkraft bzw. des Drehmoments und somit für die Steuerbarkeit des Motors. Mittels Aufstellung und Lösung eines Optimierungsproblems wurden für einen Motor mit sechs Polpaaren jeweils die optimalen Versetzungen ermittelt und zugleich gezeigt, dass mit beiden Verzahnungsarten Steuerbarkeit erreicht werden kann. Die optimalen Zielfunktionswerte sind ein Maß für die maximalen Tangentialkräfte und zeigen, dass ein schachbrettverzahnter Motor deutlich höhere Tangentialkräfte erreichen kann als ein vergleichbarer kreuzverzahnter Motor. Die erreichbaren Radialkräfte unterscheiden sich dabei nur geringfügig. Dieser Vorteil wird jedoch erkauft durch eine Verkopplung der Vorschubkräfte, was eine aufwändigere Ansteuerung schachbrettverzahnter Motoren zur Folge hat.

Weitere Untersuchungen auf diesem Gebiet sind geplant. Die Analyse der Steuerbarkeit eines schachbrettverzahnten Motors wurden an einem Beispiel mit sechs Polpaaren durchgeführt. Ein solcher Motor wurde bereits hergestellt und erfolgreich in Betrieb genommen. Zum einen soll die Verzahnung auch messtechnisch bei schwebendem Rotor untersucht werden. Zum anderen soll untersucht werden, wie weit die Anzahl der benötigen Pole und Wicklungen eines schachbrettverzahnten Motors noch reduziert werden kann, ohne die Steuerbarkeit zu beeinträchtigen. Dazu ist eine weitere Berechnung mittels des Optimierungsalgorithmus geplant sowie ggf. ein experimenteller Nachweis durch einen weiteren Versuchsaufbau. In diesem Zusammenhang soll auch auf den Nachweis der vollständigen Steuerbarkeit, welcher auch die Radialkräfte umfasst, sowie die konkrete Ansteuerung der Wicklungen eingegangen werden.

\section{Danksagung}

Der Deutschen Forschungsgemeinschaft (DFG) wird gedankt für die Förderung dieses Projektes im Rahmen einer Sachbeihilfe (GZ: WE 4347/4-1).

Open Access This article is distributed under the terms of the Creative Commons Attribution 4.0 International License (http://creativecommons.org/ licenses/by/4.0/), which permits unrestricted use, distribution, and reproduction in any medium, provided you give appropriate credit to the original author(s) and the source, provide a link to the Creative Commons license, and indicate if changes were made.

\section{Literatur}

1. Schweitzer, G., Maslen, E. H. (2009): Magnetic bearings-theory, design, and application to rotating machinery (S. 1-24). Berlin: Springer.

2. Michioka, C., Sakamoto, T., Ichikawa, O., Chiba, A., Fukao, T. (1996): A decoupling control method of reluctance type bearingless motors considering magnetic saturation. IEEE Trans. Ind. Appl., 32, 1204-1210.

3. Nezamabadi, M. M., Afjei, E., Torkaman, H. (2016): Design and electromagnetic analysis of a new rotary-linear switched reluctance motor in static mode. Appl. Comput. Electomagn. Soc. J., 31, 171-178. 2.

4. Elganai, A. A. M. (2016): Study of rotary-linear switched reluctance motor. Int. J. Eng. Trends Technol., 31, 149-152. 1.

5. Schleicher, A., Werner, R. (2017): Berührungsloses 6-Achs-Messssystem für lagerlose reluktante Rotations-Linearmotoren. In Tagungsband 11. Workshop Magnetlagertechnik Zittau-Chemnitz (S. 67-73) 5.-6. 9. 


\section{Autoren}

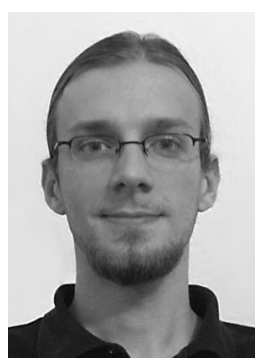

\section{André Schleicher}

absolvierte von 2008 bis 2014 das Bachelorstudium der Elektrotechnik und Masterstudium der regenerativen Energietechnik an der Technischen Universität Chemnitz, Deutschland. Von 2014 bis 2015 war er als wissenschaftlicher Mitarbeiter am Lehrstuhl für Leistungselektronik und elektromagnetische Verträglichkeit tätig. Seit 2015 ist er wissenschaftlicher Mitarbeiter am Lehrstuhl fü elektromagnetische Energiewandlungssysteme und Antriebe und hat sein Promotionsstudium zum Thema lagerloser reluktanter Rotations-Linearmotor aufgenommen.

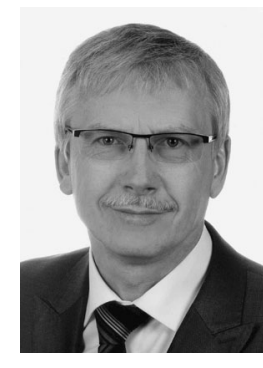

\section{Ralf Werner}

studierte Elektrotechnik und promovierte an der Fakultät für Elektrotechnik und Informationstechnik der TU Chemnitz, Deutschland. Bis zum Jahr 1994 war er als wissenschaftlicher Mitarbeiter an dieser Fakultät tätig. Anschließend arbeitete er als Projektingenieur und Projektleiter in mehreren mittelständischen Unternehmen. Seit 2009 hat er die Professur für Elektrische Energiewandlungssysteme und Antriebe an der TU Chemnitz inne. Seine Arbeitsschwerpunkte liegen auf den Gebieten der elektrischen Maschinen, der Antriebstechnik, der Leistungselektronik, der Mechatronik und dem 3D-Druck von Motorkomponenten. 